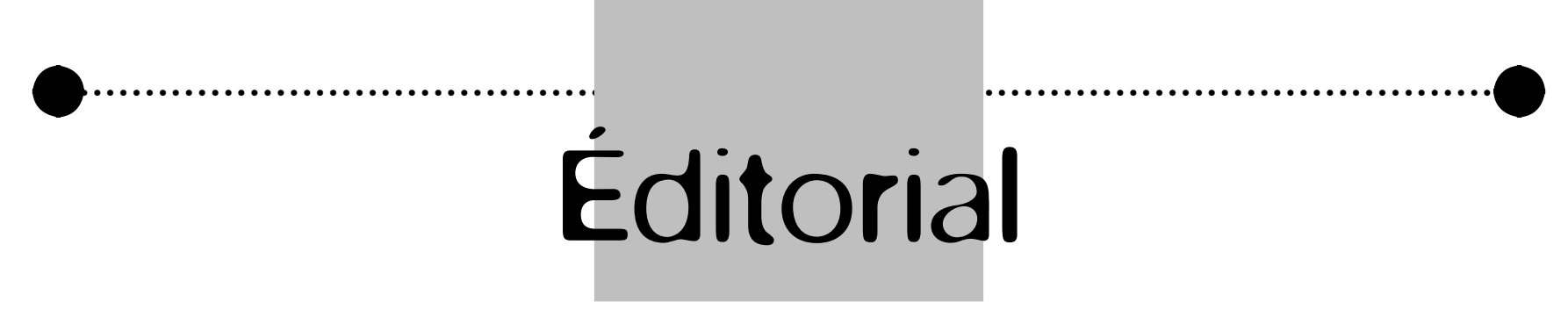

\title{
Psychiatrie et santé mentale
}

\section{Psychiatry and mental health}

Psychiatre

des Hôpitaux

Honoraire, Président

d'honneur de «Santé

Mentale France ». 121,

avenue d'Italie, 75013

Paris, France.

b.j.durand@free.fr

\author{
Bernard DURAND
}

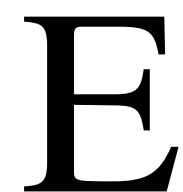

n octobre 2016, un conseil national de santé mentale a été mis en place par la ministre de la Santé, présidé par A. Ehrenberg, sociologue dont les travaux font autorité.

C'était peut-être la première fois que l'on officialisait à ce niveau le terme de santé mentale et le fait que la psychiatrie, seule, ne pouvait apporter l'ensemble des réponses nécessaires à ceux qui sont confrontés à des maladies mentales, que ce soit en termes de logement, d'insertion professionnelle ou de lien social, toutes choses que la psychiatrie a plus ou moins essayé de prendre en compte pendant longtemps du fait des représentations sociales et de la stigmatisation liées à ces maladies.

Mais les psychiatres, nostalgiques de la commission des maladies mentales, ont mal vécu que ce conseil ne soit que de santé mentale, comme si la psychiatrie risquait de s'y perdre, et encore plus que ce fut un sociologue qui préside cette instance nationale. Le changement de ministre a conduit à ce que l'on abandonne ce conseil pour une structure de pilotage de la santé mentale et cette fois-ci de la psychiatrie. Cela dit, il est évident que le temps où il s'agissait de lutter contre le fléau so- cial des maladies mentales est bien révolu. C'est dans le louable souci de lutter contre la stigmatisation que le vocabulaire a évolué et que l'on évite aujourd'hui de parler de «maladie mentale » pour lui préférer des signifiants comme souffrance psychique ou trouble mental, promu par le DSM. On entend même de plus en plus souvent l'expression «trouble de santé mentale ». Mais a-t-on déjà entendu parler de troubles de santé cardiaque ou de santé rénale ? Cela ne veut rien dire et ce n'est pas avec de tels euphémismes que l'on fera reculer la stigmatisation. Le terme de santé mentale s'est néanmoins imposé aujourd'hui dans les politiques publiques, mais sa dimension polysémique mérite d'être explicitée, car elle induit parfois une confusion entre le champ du soin individuel, la psychiatrie, et celui de la santé publique, la santé mentale, même si leur complémentarité est évidente.

C'est avec le rapport Demay, en 1982, que des psychiatres progressistes ont introduit euphémiquement le terme de santé mentale pour parler de la psychiatrie, en proposant de remplacer les hôpitaux psychiatriques, liés aux pratiques asilaires, par des établissements publics de santé mentale (EPSM). Le décret du 14 mars 1986 a ensuite créé 
les conseils départementaux de santé mentale (CDSM), bien que l'on y évoquât encore la lutte contre les maladies mentales. C'est la circulaire du 14 mars 1990 qui introduisit l'idée d'une politique de santé mentale qui dépasse les seuls soins psychiatriques. Le concept de santé mentale avait néanmoins déjà été utilisé, dès les années cinquante, au sein de la Fédération des Sociétés Croix Marine, où l'on parlait d'aide à la santé mentale à propos de l'implication du social dans la prise en charge des patients. Ce mouvement a été conçu initialement pour «apporter un secours et une entraide aux handicapés psychiques» dans un «esprit médico-social », selon la formule de son fondateur Pierre Doussinet; celui-ci affirmait ainsi que la continuité de vie des patients ne relevait pas que des soins sanitaires et cela bien avant que le médico-social ne devienne le champ spécifique de l'accompagnement des personnes en situation de handicap. Dans cette acception, la santé mentale rend compte du fait que la vie et les modalités d'aide pour les personnes confrontées à des troubles psychiques dépassent largement la question des soins psychiatriques.

À la même époque, la Ligue française d'hygiène mentale était très active sous l'impulsion de P. Sivadon et menait des actions d'information et de prévention. Celle-ci avait été créée par Édouard Toulouse dans une démarche de type hygiéniste qui correspond aujourd'hui à ce que nous appelons prévention, mais notons qu'Édouard Toulouse est aussi celui qui a ouvert les premiers lits de psychiatrie dans un autre cadre que la loi de 1838 .

Si le concept de santé mentale s'inscrit pour une part dans la continuité de ces pionniers d'un écart avec la psychiatrie proprement dite, il s'inscrit également dans la suite de la définition de la santé de l'OMS, qui fait de « la santé un état complet de bien-être physique, mental et social et non pas seulement une absence de maladie ou d'infirmité ». En 2001, l'OMS définit la santé mentale « comme un état de bien-être dans lequel une personne peut se réaliser, surmonter les tensions normales de la vie, accomplir un travail productif et contribuer à la vie de sa communauté. Dans ce sens positif, la santé mentale est le fondement du bien-être d'un individu et du bon fonctionnement d'une communauté ». La santé mentale serait donc le moyen de s'épanouir sur le double plan intellectuel et émotionnel, mais aussi de trouver et de tenir sa place dans la vie sociale, scolaire et professionnelle. Pour la société, elle contribue à la prospérité, à la solidarité et à la justice sociale. À l'inverse, la mauvaise santé mentale est pour les citoyens comme pour les mécanismes sociétaux une source de pertes, de dépenses et de charges de toutes sortes. En 2005, la conférence ministérielle européenne de l'OMS sur la santé mentale a débouché sur de solides engagements politiques et sur un plan d'action exhaustif que l'on retrouve dans «le livre vert sur la santé mentale en Europe ». Celui-ci précise que la santé mentale de la population européenne est l'un des moyens d'atteindre quelques-uns des objectifs stratégiques de l'Union européenne et d'améliorer de manière tangible et concrète la qualité de la vie des citoyens européens. Il est clair qu'il ne s'agit plus ici de la psychiatrie, mais d'axes de santé publique et même de politique tout court. On peut lire ainsi dans ce livre vert que la santé mentale permet «d'améliorer la disponibilité des ressources économiques », ce qui fait dire à certains que la santé mentale est ainsi mise en conformité avec le modèle économique néolibéral et qu'elle devient alors 
une politique de normalisation et de contrôle des citoyens.

Une autre définition de la santé mentale se retrouve dans les analyses d'A. Ehrenberg. Celui-ci a constaté que l'on emploie aujourd'hui à tout bout de champ les expressions souffrance psychique et santé mentale au point que celles-ci forment un couple solidaire, car l'une ne se comprend que dans sa relation à l'autre. Les problèmes familiaux, la souffrance au travail, les conséquences de problèmes sociaux et économiques, les échecs à répondre aux exigences des normes d'autonomie, s'expriment dans un langage qui met en relation malheur personnel et relations sociales perturbées sous le vocable de la « souffrance psychique ». Ces changements dans la manière de subir ont créé une nouvelle morbidité qui élargit considérablement le spectre des pathologies. «Nombre d'entités psychopathologiques sont devenues aujourd'hui des questions sociales, tandis qu'un nombre sans cesse croissant de questions sociales sont appréhendées au prisme des catégories et entités psychopathologiques », explique A. Ehrenberg et cela nécessite que la psychiatrie puisse être englobée dans un champ plus large qui est la santé mentale.

Le rapport Piel-Roelandt de 2001, prenant en compte les différentes composantes de la santé mentale, préconisait de mener la politique de secteur à son terme en passant de la psychiatrie à la santé mentale; à l'époque, cela avait suscité quelques remarques acerbes, alors même que ce rapport affirmait le caractère sanitaire de la psychiatrie et préconisait l'ouverture vers le médicosocial et le social.

Le dernier rapport de Michel Laforcade, en 2016, est « relatif à la santé mentale »; il préconise une politique recouvrant une approche globale de la personne dans son milieu de vie en mobilisant tous les acteurs de la société, dans des territoires de santé mentale. Les soins psychiatriques engagés dans et avec le social et «la promotion $d u$ bien-être », selon l'expression de madame Buzin, ministre de la Santé, ne sont pas contradictoires. La psychiatrie relève de la dimension singulière des soins, mais elle a besoin de collaborer avec d'autres acteurs plus axés sur l'accompagnement dans la cité pour permettre une continuité d'existence des patients (les technocrates parlent aujourd'hui de continuité des parcours). Quant à la santé mentale, c'est une question de santé publique et elle est l'affaire de tous.

La santé mentale possède également une dimension utopique qu'ont portée de nombreux psychiatres, bien avant que l'on parlât de santé mentale positive. En 1959, un an avant la circulaire de mars 1960, Bonnafé affirmait à Tours qu'il convenait « de mettre en place une structure fondée sur un territoire au sein duquel les divers moyens concourent à la protection de la santé mentale ». Un des acteurs les plus actifs dans la mise en place de la sectorisation, Henri Duchêne, soulignait l'intrication extrême des activités proprement psychiatriques avec celles de prophylaxie et d'hygiène mentale en mettant en avant les expériences d'Amsterdam et de Nottingham qui avaient déjà lié ces deux dimensions. Et déjà, bien avant les travaux d'Alain Ehrenberg, il notait que « l'obligation de prendre en charge tous les problèmes de santé mentale conduisait en effet inévitablement à des cas se rapprochant de plus en plus de la "normale”. Or, ceux-ci, loin d'être plus simples à traiter, requièrent au contraire infiniment plus de temps spécialisé tant du psychiatre que de ses collaborateurs, que la pathologie men- 
tale majeure ». Un autre psychiatre de cette génération, Pierre Bailly-Salin, dans un élan utopiste caractéristique de celle-ci, proposait comme définition de l'hygiène mentale «la médecine $d u$ bonheur », tout en notant qu'une telle formule ne résolvait pas le problème qui reste toujours entier. Et il ajoutait : «le psychiatre d'hygiène mentale sera-t-il le médecin $d u$ bonheur $d u$ monde ou le médecin du bonheur de l'individu?».

\section{LIENS D'INTÉRÊT}

L'auteur déclare n'avoir aucun lien d'intérêt concernant les données publiées dans cet article.

\section{Communiqué}

\section{COLLOQUE FRANCO-JAPONAIS}

Sous l'égide de l'Ambassade de France au Japon

PROGRAMME SCIENTIFIQUE

\section{SÉMINAIRE À L'UNIVERSITÉ DE NAGOYA :}

Le 5 novembre 2019 : Psychopathologie de l'hikikomori : aspects sociologiques, familiaux, psychologiques et thérapeutiques. Prs et Drs Suzuki, Furuhashi, Benhamou, Guedj-Bourdiau

\section{COLLOQUE À LA MAISON FRANCO-JAPONAISE DE TOKYO :}

Le 8 novembre 2019 : Cardiologie médicale et chirurgicale :

- Pr Kario (département de médecine cardio-vasculaire de l'université médicale Jichi)) : l'hypertension artérielle au Japon.

- Pr Blacher (hôpital de l'hôtel-Dieu à Paris, past-président de la Société Française de l'Hypertension artérielle) : nouvelles données épidémiologiques françaises en matière d'hypertension artérielle.

- Pr Nataf, (service de chirurgie cardio-vasculaire de l'hôpital Bichat à Paris) : thème à préciser.

- Pr Aomi,(département de chirurgie cardiovasculaire de l'Université Médicale pour femmes de Tokyo, directeur du centre Marfan et aorte de l'hôpital vasculaire Ayasé) : thème à préciser.

Le 9 novembre 2019 : Neurobiologie et psychiatrie : Addictions.

- Dr Benhamou (Hôpitaux de Saint Maurice) : introduction : dépendance psychique et addictions.

- Pr Tassin (Collège de France) : aspects neurobiologiques des addictions.

- Dr Kazakevicius (Centre de Santé Mentale Angevin) : traumatismes sexuels précoces et comportements addictifs sexualisés dans l'enfance. Réflexions à partir du cas d'une enfant et de ses soins psychiques.

- Dr Thomas Wallenhorst (Centre hospitalier de Semur-en-Auxois) : l'hôpital de jour : un nouvel outil en addictologie.

- Dr Delphine Hochart : troubles des conduites alimentaires et addiction au sport.

- Dr Higuchi (Centre Médical National pour les Addictions Kurihama) : addiction à l'internet au Japon.

- Dr Noma (Département de psychiatrie,Université de Kyoto) : troubles addictifs du comportement alimentaire : la boulimie au Japon

- Pr Takahashi (Département de psychiatrie, Université Médicale et Dentaire de

Tokyo) : addictions et neuro-imagerie.

Pour toute information et inscription :

s'adresser au Dr Hervé Benhamou

email : hbenhamou@aol.com 